\title{
Computer Simulation Based Optimization of Aspect Ratio for Micro and Nanochannels
}

\author{
Muhammad Javaid Afzal ${ }^{1}$, Muhammad Waseem Ashraf ${ }^{2}$, Shahzadi Tayyaba ${ }^{3}$ \\ Akhtar Hussain Jalbani ${ }^{4}$, Farah Javaid ${ }^{5}$
}

RECEIVED ON 05.09.2018, ACCEPTED ON 26.07.2019

\begin{abstract}
For Computational Fluidic Dynamics (CFD) analysis, different types of software are available such as ANSYS, COMSOL, GAMBIT and ABAQUS. ANSYS FLUENT is a very influential CFD software that enables the researchers to execute advanced and fast simulation as the performance of product is optimized. In this research work, the capabilities of ANSYS FLUENT have been investigated through the fluidic simulation of nano scale channels. Here, the simulation of three straight shaped and one curvilinear nano channel has been performed to analyze the precision of simulation with the aspect ratio of smaller dimensions (radius, length). The accurate dimensions have been determined for various shapes of channels. For straight circular channel, the radius of $124.9 \mu \mathrm{m}$ and length of $900 \mu \mathrm{m}$ are the dimensions found for error free simulations with 7.20 aspect ratio. While for straight crossed channel, the radius of $5.1 \mathrm{~mm}$ and length of $100 \mathrm{~mm}$ are the accurate dimensions for error free simulations with 19.60 aspect ratio. Width and depth are determined as $180 \mu \mathrm{m}$ with $180 \mu \mathrm{m}$ length in case of straight square channel for error free simulations with 1.0 aspect ratio. Finally, in case of curvilinear channel for error free simulations, the radius and length are found 22 and $209.1 \mu \mathrm{m}$ respectively with 9.50 aspect ratio. None of the channels is found fit for simulation in nanometer range. Even in micrometer range, the simulations of these channels have acquired errors. Moreover, it has been observed that the aspect ratio is different for different geometries of microchannels for error free simulation.
\end{abstract}

Keywords: Computational Fluid Dynamics, ANSYS, Microchannels, Nanochannels, Simulations.

\section{INTRODUCTION}

CFD is the use of applied mathematics, physics and computational software to visualize the flow of liquid. CFD analysis presented a complex, fast, effective simulation of transfer of heat, mixing, unsteady and compressible fluid flows [1-2]. Through CFD analysis, the fluid forces can be determined and performance of impact of a liquid or gas can easily be understood. Gas and fluid flow with transfer of heat, internal and external flow of the liquid, turbulent, laminar, and transitional flows, gas and fluid mixing, viscous and non-viscous fluid, real and compressible gas, non-Newtonian fluids like blood, honey, molten plastics are the aspects and applications of CFD [3-6]. Through simulations calculations are made by the computers with the help of boundary conditions. ANSYS, COMSOL, GAMBIT, ABAQUS are the software packages for the analysis of the fluid flow [7]. Amongst all of them ANSYS is a very powerful tool for analysis of fluid flow. It is an American Computeraided engineering finite elemental analysis software. It is a CFD tool with incredible flexibility and accuracy with widespread applications. It goes beyond the wellvalidated CFD qualitative results to provide fast precise numerical predictions for fluid flow. ANSYS

\footnotetext{
${ }^{1}$ Department of Physics, Government Islamia College, Civil Lines, Lahore, Pakistan. Email: javaidphy@ gmail.com (Corresponding Author)

${ }^{2}$ Department of Physics (Electronics), GC University, Lahore, Pakistan. Email: Muhammad.waseem.ashraf@gmail.com

${ }^{3}$ Department of Computer Engineering, The University of Lahore, Lahore, Pakistan. Email: shahzadi.tayyaba@ hotmail.com

${ }^{4}$ Department of Computer Science, Quaid-e-Awam University of Engineering and Technology. Email: jalbaniakhtar@ gmail.com

${ }^{5}$ Department of Physics, Government APWA College, Lahore, Email: farahjavaid71@ gmail.com

This is an open access article published by Mehran University of Engineering and Technology, Jamshoro, under CC BY 4.0 International License.
} 
can be used to innovate with breakthrough capabilities in microfluidic devices such as microchannels, micropumps and microneedles. CFD analysis through ANSYS is used in microchannels research like convective heat transfer, validation of microchannel cooling, heat transfer, pressure loss measurements, flow characteristics and micromixing modelling [8-9]. The overall consequence of aspect ratio on fluid flow in a micro/nano-channel is examined by ANSYS in this paper. Beforehand, the literature has the investigations for the flow parameters on geometrical parameters on micro/nano-channels. But in this study, the authors have simulated micro/nano-channels for better fluid flow. The capability of ANSYS software has been checked extensively. Here, the effect of aspect ratio has been explained by varying the diameter and the length of the channels.

CFD analysis of straight channels cannot be done without the aspect ratio. It is the ratio of maximum dimension to minimum dimension of the channel used. It is a significant factor in flow analysis for microchannels. Rosaguti et al. [10] used ANSYS CFX-10 for sinusoidal microchannels to study heat transfer at low Reynold numbers. They used very low aspect ratio i.e. 4.5 for CFD analysis. Lee et. al. [11] examined the influence of the aspect ratio on rectangular microchannels. Ashraf et. al. [12] used aspect ratio with $160 \mu \mathrm{m}$ length and $15 \mu \mathrm{m}$ diameter for cell sorting system through sinusoidal microchannel. Tayyaba et. al. [13] used high aspect ratio with 1600 and $15 \mu \mathrm{m}$ diameter for cell sorting system through sinusoidal microchannel. Solehati et. al. [14] used high aspect ratio with 10,000 $\mu \mathrm{m}$ length and $500 \mu \mathrm{m}$ for mixing performance in $\mathrm{T}$-junction sinusoidal microchannel. Ozkan and Erdem [15] used COMSOL Multiphysics software to study the mixing performance in sinusoidal microchannels with aspect ratio of $100 \mu \mathrm{m}$ length and $0.5 \mathrm{~nm}$ diameter. Chiam et. al. [16] used Solidworks and ANSYS with the aspect ratio of 2 for investigation of heat transfer through sinusoidal microchannel. Mixing performance in $\mathrm{T}$ junction microchannel has been investigated to study the effect of the aspect ratio for flow analysis, fluid velocity and mixer microchannel [17-19]. Nivedita et. al. [20] used low aspect ratio for studying the Dean flow dynamics through spiral channels. Damian et. al. [21] examined three crossed microchannels with their aspect ratios. Afzal et. al. [22] studied the American Society of Military Comptrollers (ASMC) and Department of Sacred Music \& Communications (DSMC) successfully with the help of ANSYS Fluent. Kwak et. al. [23] have studied the heat flux from microchannels by using high aspect ratio. Previously and presently, all the researchers working on CFD have done the ANSYS simulations on individual element [24-25]. The usage of a computer to characterize the fluid dynamics in micro/nanochannels and in other fields is wide spread [26-29].

\subsection{Mesh Dependence}

Mesh dependency is used for the following steps for every simulation. For fine mesh analysis, relevance is set at 0 . The size function is set at curvature. Fine relevance center is selected. Maximum face size is set at $0.38205 \mathrm{e}-003$. Inflation option is set at smooth transition. First simulation has been run with initial mesh with the confirmation of convergence. Once the convergence criteria were selected for the initial simulation, mesh has been improved globally. Because the solution varies with the modification of mesh, the solution is mesh dependent. Hence mesh dependency was engaged.

\section{ANSYS FLUENT SIMULATION FOR NANOCHANNELS}

In this research work, straight circular, straight crossed, straight square and curvilinear nanochannels were investigated extensively through ANSYS FLUENT simulations. From the function of "Create/Edit materials" water was selected as the flowing fluid from the "Fluent Database" in the set up tool of "ANSYS FLUENT". Then boundary conditions were set with $0.1 \mathrm{~m} / \mathrm{s}$ velocity and $100 \mathrm{~Pa}$ pressure. These conditions were kept for all simulations of each and every size of nanochannels.

\subsection{Straight Circular Nanochannel}

First of all, in design modeler micrometer unit was selected because there was no smaller unit available than this. The units available were meter, centimeter, millimeter, micrometer, inch and foot. If a nano size element is to be created in design modeler, then there 
is a compulsion in ANSYS to opt micrometer as a unit. Because there is no nano unit manufactured in ANSYS, therefore, for nano size $0.001 \mu \mathrm{m}$ was used as $1 \mathrm{~nm}$. One could use even lower than $1 \mathrm{~nm}$ by selecting this scale in ANSYS FLUENT. Although less than $3 \mathrm{~nm}$ is not available in some versions of ANSYS. Straight circular nanochannel was designed in design modeler and meshed in mesh analysis. The number of nodes and elements were increased and decreased in mesh analysis by varying the relevance between 0-100 for better mesh. It could also be enhanced by varying minimum size, maximum size and maximum face size of the element used in sizing tool. In sizing tool of mesh analysis relevance center and smoothing were set at fine and high respectively. Also, the function "Use Advanced Size Function" was set on curvature. The geometry of the channel was created in design modeler and meshed in mesh analysis which is shown in Fig. 1.

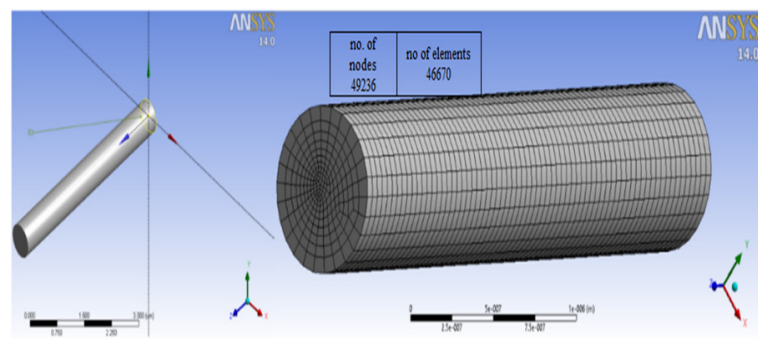

Fig.1: Straight Circular Nanochannel with Design Modeler and Mesh Analysis

After setting of "Reference Values", "Hybrid Initialization" method was adopted in the "Solution Initialization" tool from setup. During simulations different errors occurred. The errors "Divergence detected in AMG solver: Pressure correction" (accuracy for radius up to $300 \mathrm{~nm}$ and length up to 3 $\mu \mathrm{m})$, "Divergence detected in AMG solver: $\mathrm{x}$ momentum" and before them a warning error "Warning: convergence tolerance of 1.000000e-6 not reached during Hybrid Initialization" occurred when straight circular nanochannel was considered. If pressure and velocity were changed to get rid of the error of "Divergence detected in AMG solver: Pressure correction" then the error of "Divergence detected in AMG solver: $x$-momentum" occurred and vice versa. These errors could also have occurred in bigger channels when there was wrong shape of geometry, wrong mesh and wrong boundary conditions (Fig. 2(a-c)).

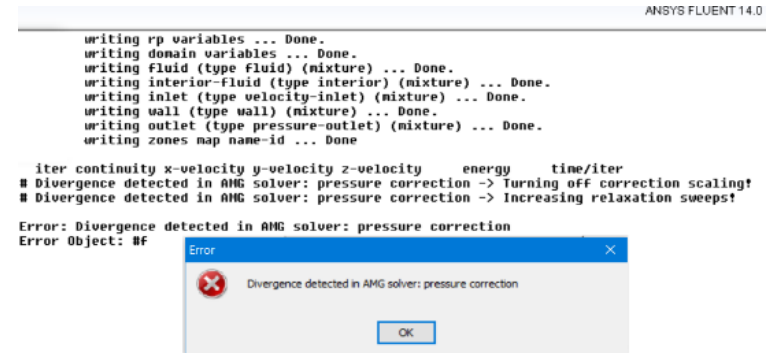

Fig. 2(a): Pressure Correction Error

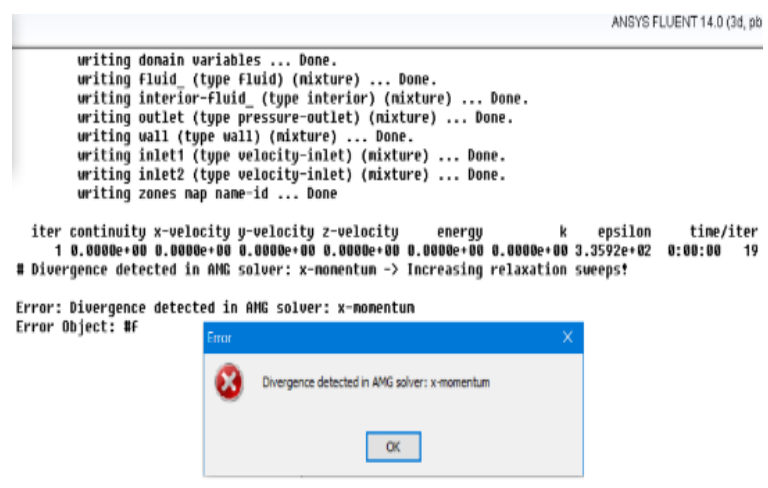

Fig. 2(b): X-Momentum Error

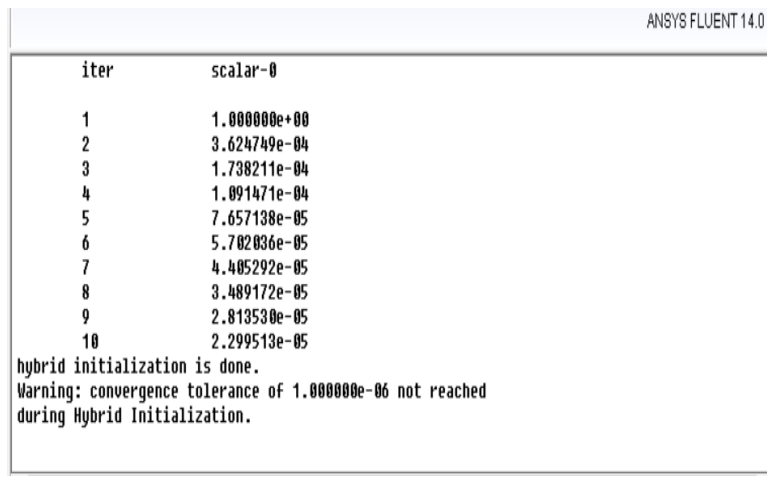

Fig. 2(c): Warning Error

With these errors, there were no simulations. Actually, these are the errors of ANSYS which cannot handle the nano size element. When the dimensions of the channel were increased up to $300 \mathrm{~nm}$ radius and 3.1 $\mu \mathrm{m}$ length, with the same boundary conditions then these errors were eliminated automatically and simulation was performed with the same warning error "Warning: convergence tolerance of 1.000000e-6 not reached during Hybrid Initialization" that appeared in setup tool of ANSYS. The contour of pressure for this simulation is shown in Fig. 3.

With the warning error, the received data had first five zero iterations from the total iterations. This type of 


\section{Computer Simulation Based Optimization of Aspect Ratio for Micro and Nanochannel}

warning could be ignored. Then flow velocity, flow rate and other parameters could be determined easily from the obtained data. The first five zero iterations can be observed in Fig. 4.

When radius was decreased from 300-299.9 nm, a new error " 1 solution is converged" occurred. Simulation was not done with this error. When the size was more increased from nano size to $71.1 \mu \mathrm{m}$ radius and 400.1 $\mu \mathrm{m}$ length then warning error was eliminated with the same boundary conditions and simulation was not done. This time the error was "2 solution converged". At $80 \mu \mathrm{m}$ radius and $500 \mu \mathrm{m}$ length, the error "3 solution is converged" occurred. Still no simulation was done. The error "4 solution is converged" occurred at $100 \mu \mathrm{m}$ radius with $500 \mu \mathrm{m}$ length. Finally, the error "5 solution is converged" occurred up to $124.8 \mu \mathrm{m}$ with $900 \mu \mathrm{m}$. These errors are shown in Fig. 5(a-e).

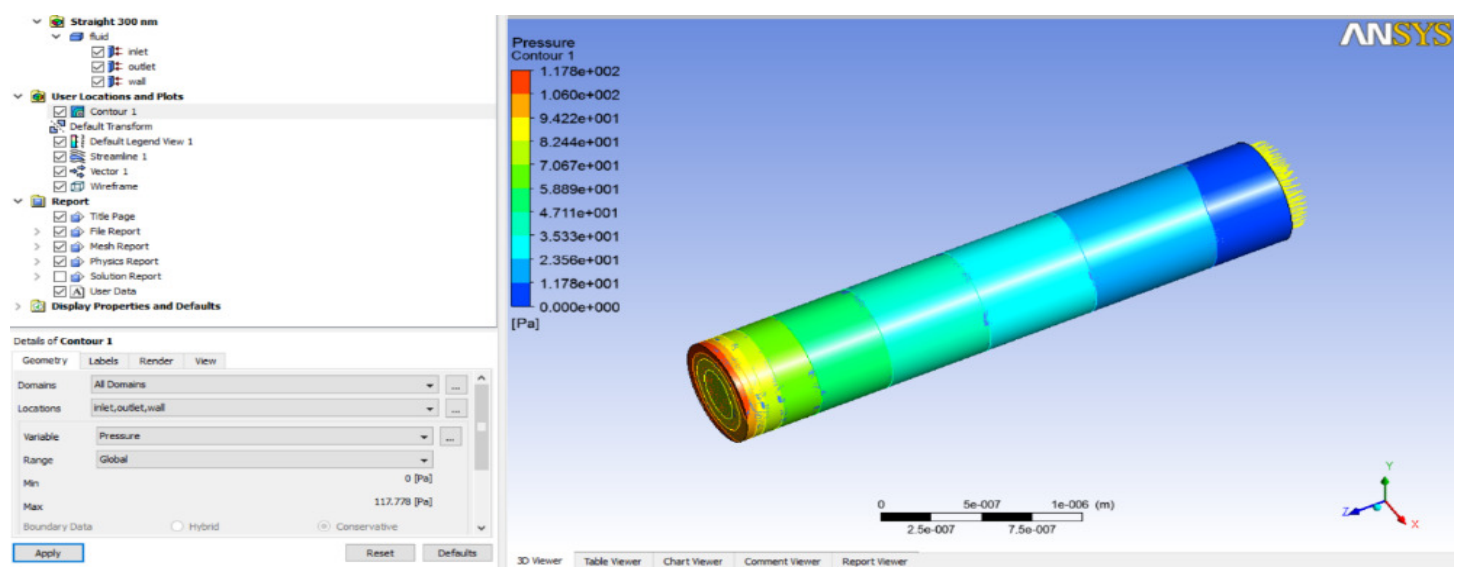

Fig. 3: Control of Pressure for $300 \mathrm{~nm}$ radius and $3.1 \mathrm{~mm}$ length straight circular channel

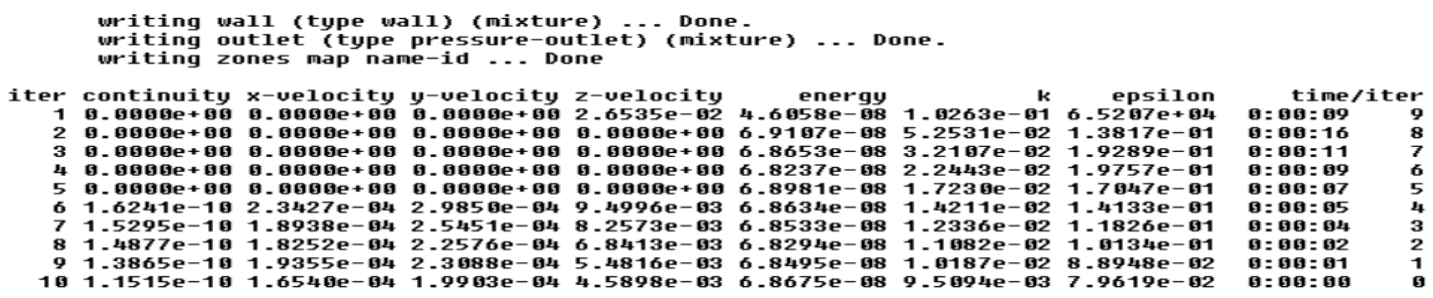

Fig. 4: First five zero iterations from total iterations

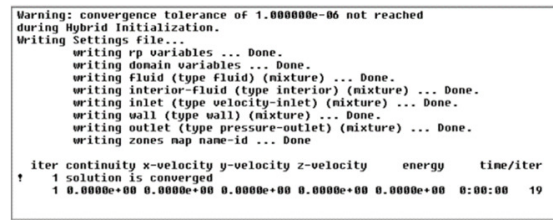

Fig. 5(a): 1-Solution is converged

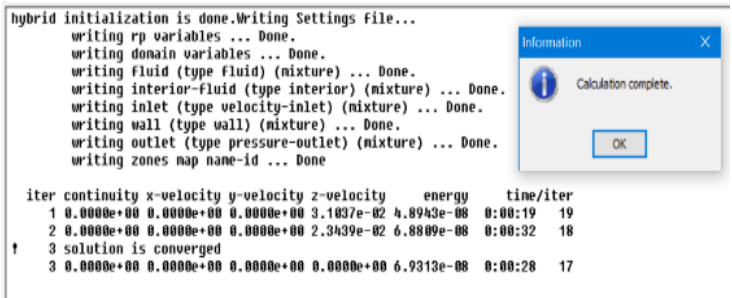

Fig. 5(c): 3-Solution is converged

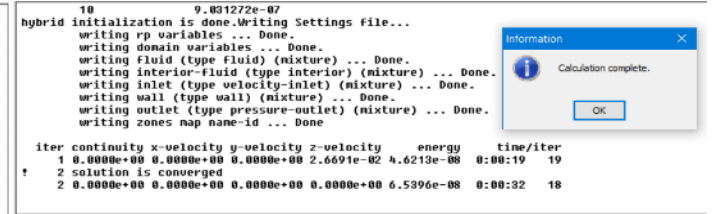

Fig. 5(b): 2-Solution is converged

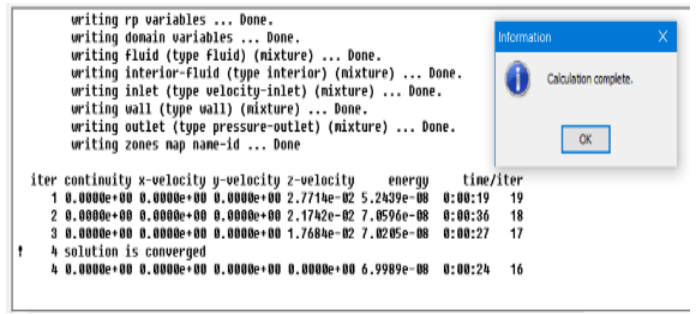

Fig. 5(d): 4-Solution is converged 


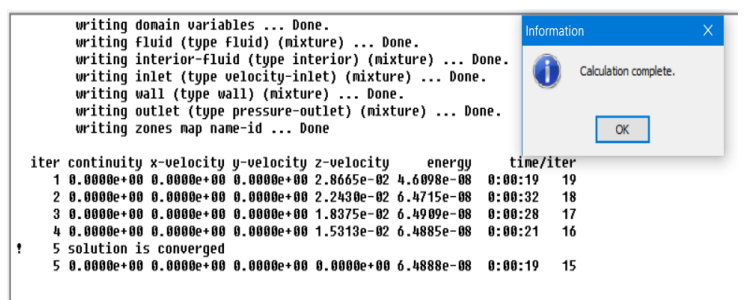

Fig. 5(e): 5-Solution is converged

These errors meant that the fluid could not enter the channel and flow was converged. Fluid could not pass through the channel. When the size was adjusted above or equal to $124.9 \mu \mathrm{m}$ radius and $900 \mu \mathrm{m}$ and error free simulation was done at this range. When length was readjusted up to $899.9 \mu \mathrm{m}$ then again simulation was not done. The contour of pressure for this simulation is shown in Fig. 6 .

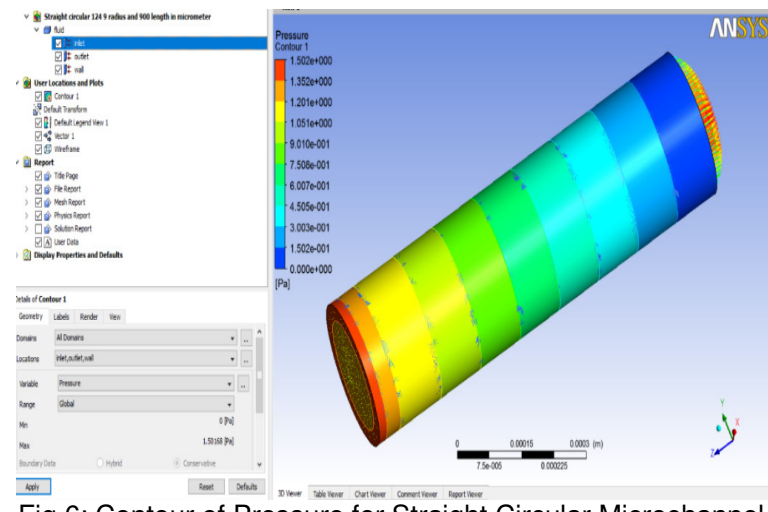

Fig.6: Contour of Pressure for Straight Circular Microchannel

With warning error simulation was done for straight circular microchannel at 103.3 aspect ratio. Error free simulation was done for this microchannel at 7.20 aspect ratio. It means ANSYS works best with these dimensions for straight circular microchannel. The summary of the simulated data is shown in Fig. 7. The errors are along $\mathrm{y}$-axis and dimensions are along $\mathrm{x}$ axis.

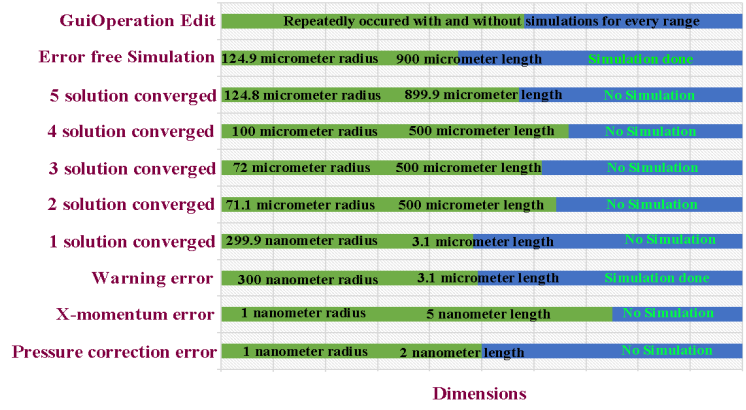

Fig. 7: Graphical Summary of Straight Circular Nanochannel

\subsection{Straight Crossed Nanochannel}

The name straight crossed was given to this type of nanochannel because of two straight perpendicular nanochannels. This nanochannel was created in nano size and meshed in design modeler and mesh analysis respectively. The number of nodes and elements were varied in mesh analysis by changing the relevance between 0-100 for accurate mesh. It could also have enhanced by varying minimum size, maximum size and maximum face size of the element used in sizing tool. In sizing tool of mesh analysis relevance center and smoothing were set at fine and high respectively. Also, the function "Use Advanced Size Function" was set on curvature. Figu 8 from design modeler and mesh analysis for this nanochannel are shown in Fig. 8(a-b).
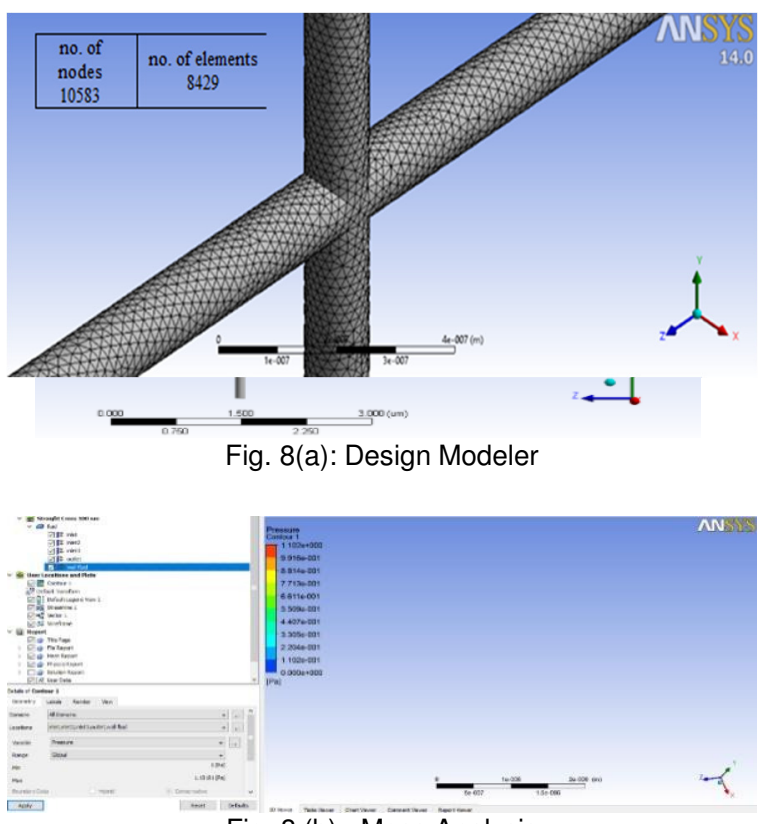

Fig. 8 (b): Mess Analysis

The dimensions of straight crossed nanochannel for the simulation has been started from $1 \mathrm{~nm}$ radius and $2 \mathrm{~nm}$ length. In nano meter range there was no simulation up to $54 \mathrm{~nm}$ radius for straight crossed nanochannels because of errors occurred in the simulation. The setup tool of the ANSYS was not accepting such a small size and giving the errors "Divergence detected in AMG solver: Pressure correction" (accuracy for radius up to $55 \mathrm{~nm}$ and length up to $0.99 \mu \mathrm{m})$, "Divergence detected in AMG solver: x-momentum" and "Warning: convergence tolerance of 1.000000e-6 not reached during Hybrid Initialization" as discussed in above section. Same 
boundary conditions were kept for this channel and similar changes were done but no simulation took place. The number of nodes and elements were changed many times in mesh analysis but all in vain and no simulation observed. Simulation was done only at $54.9 \mathrm{~nm}$ radius and $1 \mu \mathrm{m}$ length with the warning and i.e. "Warning: convergence tolerance of $1.000000 \mathrm{e}-6$ not reached during Hybrid Initialization". We can ignore this type of warning because of the data obtained. With this data all flow parameters could be determined and tested experimentally. In micrometer range the simulation was done with the same warning.

The number of nodes and elements were changed many times in mesh analysis but warning was there all the times. A strange type of error occurred for 100$999.9 \mathrm{~nm}$ radius with $100 \mathrm{~mm}$ length. In this error there was no mesh creation but still simulation was done. Actually these were abnormal dimensions for this channel. ANSYS should not have done the simulation but simulation was done. That error is shown in Fig. 9(a-d).

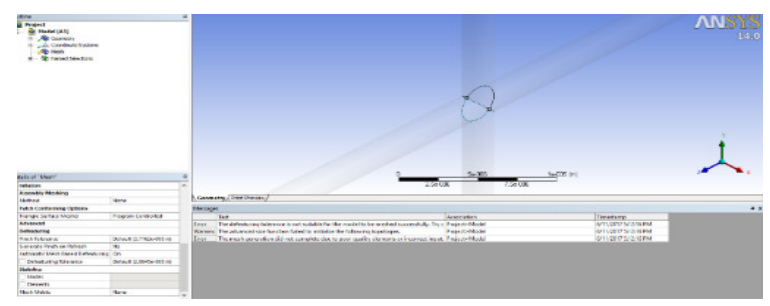

Fig. 9 (a): After meshing number of nodes and elements. Boxes are empty

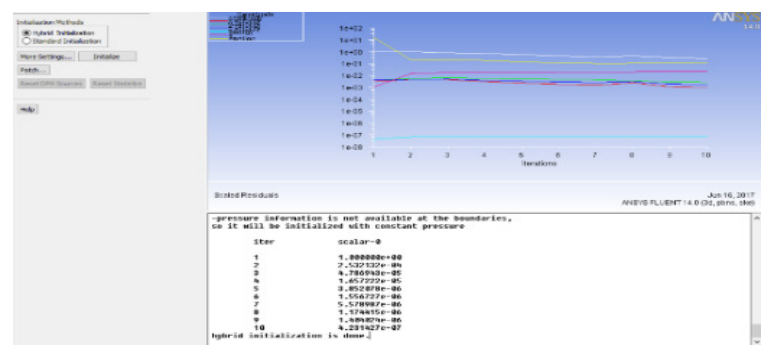

Fig. 9 (b): Initial Hybridization done without meshing

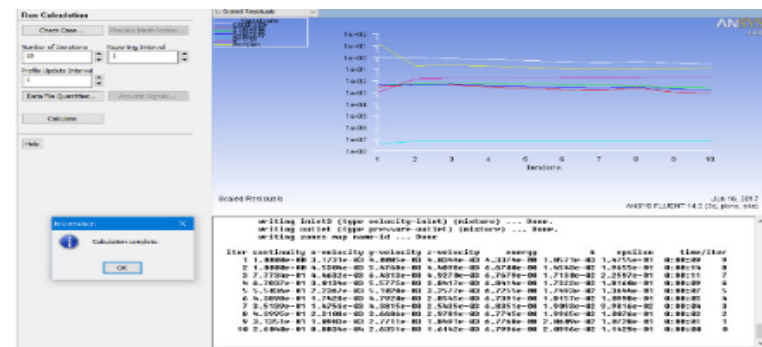

Fig. 9(c): Iterations were done

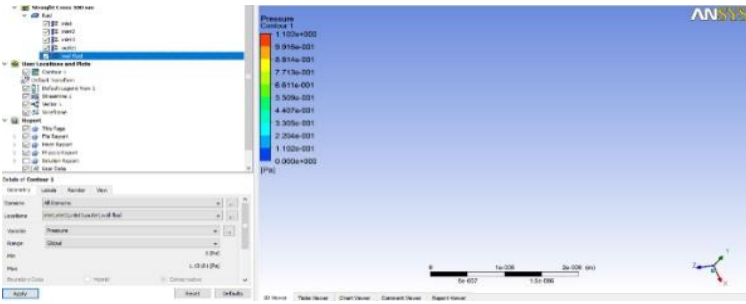

Fig. 9(d): Contour of Pressure obtained without channel diagram

When we further increased radius and length in millimeter range at $5.1 \mathrm{~mm}$ radius and $100 \mathrm{~mm}$ length simulation was done without any error with the same boundary conditions. The contour of pressure for this simulation is presented in Fig. 10.

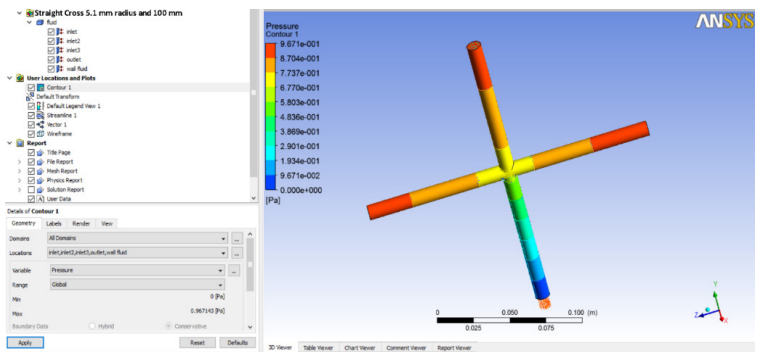

Fig. 10: Contour of pressure for simulation

With warning error simulation was done for straight crossed microchannel at 182.15 aspect ratio. Error free simulation was done for this microchannel at 19.6 aspect ratio. It means ANSYS work best with these dimensions and beyond for straight crossed microchannel. Summary of the data of each simulation is shown in Fig. 11.
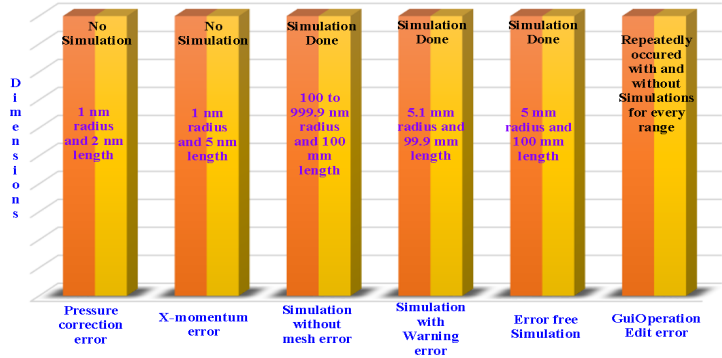

Fig. 11: Graphical summary of simulation for straight crossed nanochannel

\subsection{Straight Square Nanochannel}

Straight square nanochannel was planned in design modeler and mesh analysis. For better mesh, the 
number of nodes and elements were changed in mesh analysis by changing the relevance between $0-100$. It could also be varied by changing minimum size, maximum size and maximum face size of the element used in sizing tool function of mesh analysis. In this function of mesh analysis relevance center and smoothing were set at fine and high respectively. Here, the function "Use Advanced Size Function" was also set on curvature. Design and mesh analysis for this nanochannel are shown in Fig. 12(a-b).

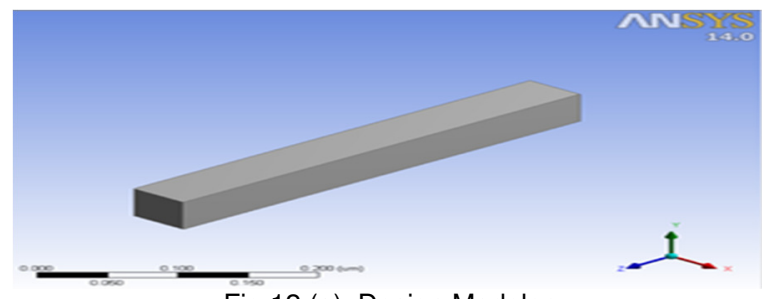

Fig 12 (a): Design Modeler

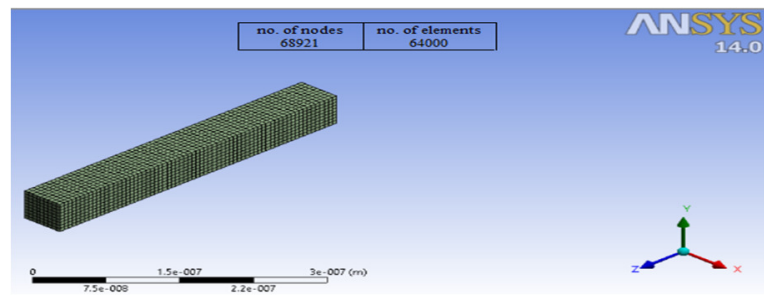

Fig. 12 (b): Mess Analysis

This channel was square in shape from inlet and outlet. The simulation for this channel started for the dimensions $1 \mathrm{~nm}$ (width and depth) and $10 \mathrm{~nm}$ in length. The error "Divergence detected in AMG solver: pressure correction" came up to $180 \mathrm{~nm}$ (width and depth) and $400 \mathrm{~nm}$ in length. If pressure was changed for correction, then the error changes to "Divergence detected in AMG solver: x-momentum". Therefore, no simulation was done. Only at $50 \mathrm{~nm}$ (width and depth) and $500 \mathrm{~nm}$ length, the error "1 solution is converged" came with no simulation. It is shown in Fig. 4 previously. If width and depth were increased up to $180.1 \mathrm{~nm}$ and still the length is $400 \mathrm{~nm}$. Simulation was done with warning error "Warning: convergence tolerance of $1.000000 \mathrm{e}-6$ not reached during Hybrid Initialization". With this warning first five iterations were obtained zero. But this error can be ignored because measurements of flow could be determined. Then length is decreased in order to obtain the minimum length for this channel for simulation. Hence, $398.2 \mathrm{~nm}$ length was obtained with the same value of width and depth and simulation was done.
Therefore, it was concluded that minimum width and depth were 180.1 and $398.2 \mathrm{~nm}$ length was suitable for simulation. There would be no simulation found below these dimensions. Warning error was still there. When the width and depth were increased up to $180 \mu \mathrm{m}$ with the $180 \mu \mathrm{m}$ length. There would be error free simulation. The contour of pressure for this simulation is shown in Fig. 13.

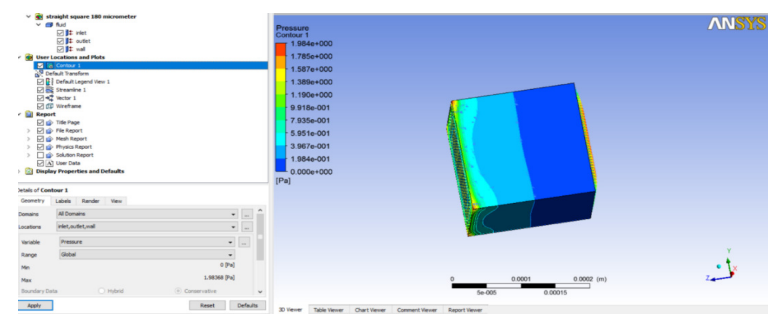

Fig. 13: Contour Pressure for Simulation

With warning error simulation was done for straight square microchannel at 2.21 aspect ratio. Error free simulation was done for this microchannel at 1.0 aspect ratio. Simulation was done beyond these dimensions without error. It means ANSYS work best with these dimensions and beyond for straight square microchannel. The summary of simulated data is shown in Fig. 14.

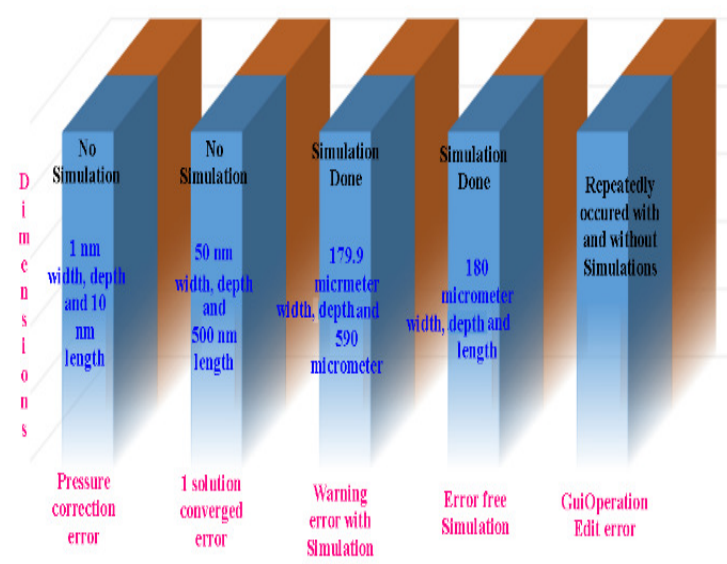

Fig. 14: Graphical summary of simulation for straight square micro/nanochannel

\subsection{Curvilinear Nanochannel}

Curvilinear channel is the channel in which there are at least two curves of the same radius. The motion of the fluid in that channel is in fixed curves and is called curvilinear motion. This nanochannel was also created and meshed in ANSYS design modeler and mesh analysis respectively. That is shown in Fig. 15(a-b). 


\section{Computer Simulation Based Optimization of Aspect Ratio for Micro and Nanochannel}
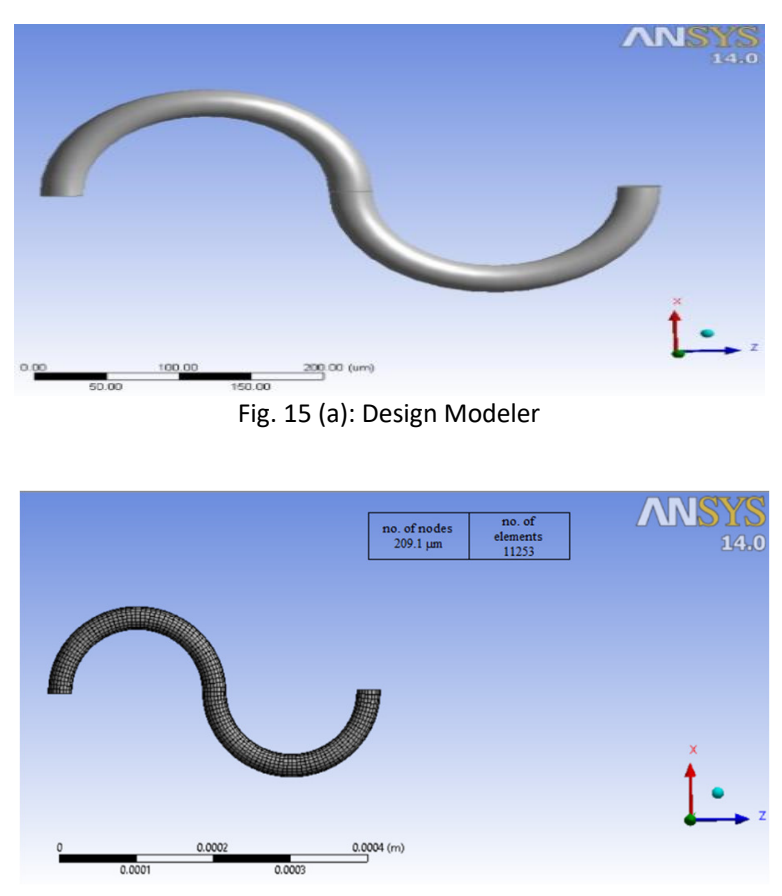

Fig. 15 (b): Mesh Analysis

The process of simulation started with channel radius 1 and $200 \mathrm{~nm}$ length. In mesh analysis the relevance was set at zero, relevance center was set at fine and smoothing was set at high. In model function of set up tool energy equation was checked. In the same function laminar viscous model and k-epsilon function was opted. From the materials tool water was selected as flowing fluid from the fluent database. Same boundary conditions and reference values were set as before used in this study. There was no simulation for $1-50 \mathrm{~nm}$ radius due to errors like "Divergence detected in AMG solver: pressure correction" and "Divergence detected in AMG solver: x-momentum" (accuracy for radius up to 50-180 nm and length up to 1.2-2.1 $\mu \mathrm{m}$ ). When radius was increased slightly to $50.1 \mathrm{~nm}$ then simulation was done with a warning error "Warning: convergence tolerance of $1.000000 \mathrm{e}-6$ not reached during Hybrid Initialization". The length was determined for the simulation and it was found 829 $\mathrm{nm}$. The simulation was done with the same warning. Then radius and length was further increased for the error free simulation. Finally, the radius and length were found 22 and $209.1 \mu \mathrm{m}$ respectively. Before these values of radius and length, simulation was done with the warning error. The contour of pressure for this simulation is shown in Fig. 16.

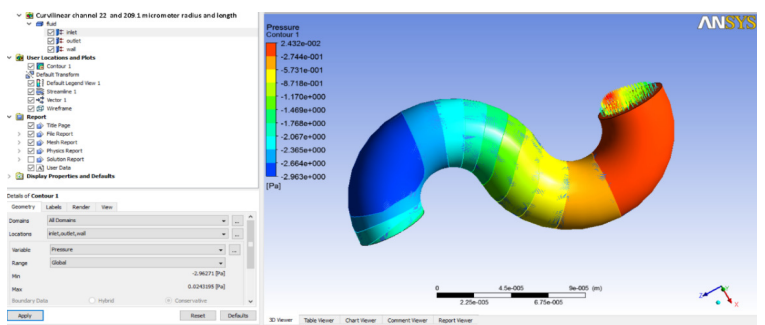

Fig. 16: Contour of pressure for simulation

Error free simulation was done for curvilinear microchannel at 9.50 aspect ratio. At these values and beyond error free simulation was done. The summary of this simulation in shown in Fig. 17.

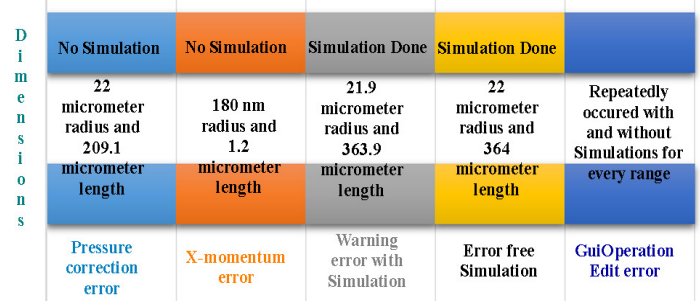

Fig. 17: Graphical summary of simulations for curvilinear Micro/Nanochannel

In this research work, a new type of error occurred again and again. Numerous simulations were done in this study again and again for longer duration of time. Therefore, ANSYS software gave the error "GuiOperation Edit". The error came when we closed design modeler and opened mesh analysis. This error also came when we closed mesh and opened set up. The error message is shown in Fig. 18.

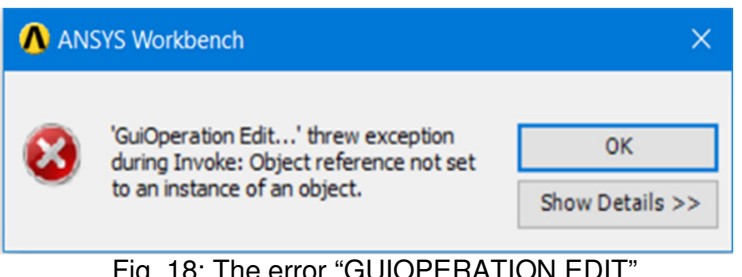

All ANSYS applications must be terminated for the elimination of above said error. There was a file in ANSYS folder named as v140 in local disk (c) of the computer. In this file there were all the ANSYS workbench configuration files. Due to the presence of these large number of files ANSYS worked slowly. We just had to rename it. This file was renamed from v140-140.old. Now all the configuration files in Workbench was hidden due to this technique. Then

Mehran University Research Journal of Engineering and Technology, Vol. 39, No. 4, October 2020 [p-ISSN: 0254-7821, e-ISSN: 2413-7219] 
ANSYS Workbench got the fresh start. New and fresh set of configuration files were generated this time. This technique worked best until the large number of files in ANSYS were built again. There is no permanent solution of this error. This error remains in the simulations. The supported graphics card and updated driver proved very necessary for the computer system to do the simulations.

\section{RESULTS AND DISCUSSION}

After all this long simulation work we have to summarize all the research work. In this novel research work, the software ANSYS is investigated in nano sized channels. We have considered straight channels and a curvilinear channel for the investigations. The errors "Divergence detected in AMG solver: pressure correction", "Divergence detected in AMG solver: $\mathrm{x}$ momentum", "1 solution is converged", "GuiOperation Edit" and "Warning: convergence tolerance of 1.000000e-6 not reached during Hybrid Initialization" are common in simulations. Amongst these errors "Divergence detected in AMG solver: pressure correction" and "Divergence detected in AMG solver: x-momentum" occurred due to the nano sized channels. ANSYS just cannot handle these sizes. In "units" function of design modeler tool, nano meter is not mentioned. The lowest unit used there is micrometer. There are 18 versions of ANSYS in market but there is no nanometer unit available in it. Therefore, ANSYS is not suitable for nano sized channels. However, nano sized object can be created in design modeler and also meshed in mesh analysis. The results are discussed below for each microchannel separately.

\subsection{Simulation of Straight Circular Micro Nanochannel}

It is found that there are limits for simulation in case of straight circular channel. The simulation of straight circular channel cannot be done when its radius and length are less than $299.9 \mathrm{~nm}$ and $3 \mu \mathrm{m}$. It means that if we take length in nano meter range then no simulation takes place. Therefore, simulation cannot be done in nano sized straight circular channel. Simulation takes place when we increase radius and length up to $300 \mathrm{~nm}$ and $3.1 \mu \mathrm{m}$ with one error and i.e. "Warning: convergence tolerance of 1.000000e-6 not reached during Hybrid Initialization". This warning is ignorable because all the measurements of fluid flow can be determined with this simulation. It works for $124.9 \mu \mathrm{m}$ radius and $900 \mu \mathrm{m}$ length without error. This means that complete range of micrometer is not suitable for simulation without error.

\subsection{Simulation of Straight Crossed Micro/Nanochannel}

In case of straight crossed microchannel simulation was done only at $54.9 \mathrm{~nm}$ radius and $1 \mu \mathrm{m}$ length with the error of warning. No simulation takes place for the values lower than $54.9 \mathrm{~nm}$ radius and $1 \mu \mathrm{m}$ length. The errors shown by the ANSYS are "Divergence detected in AMG solver: pressure correction" and "Divergence detected in AMG solver: $x$-momentum". When radius is increased up to $999.9 \mu \mathrm{m}$ then simulation was done without meshing. All the values were obtained without channel figure in the "result" tool of the ANSYS. Therefore, this simulation was not trusted. With the further increase in radius and length up to millimeter range (5.1 $\mathrm{mm}$ radius and $100 \mathrm{~mm}$ length), then error free simulation was done. It means ANSYS has problems in micrometer and millimeter range in some microchannels.

\subsection{Simulation of Straight Square Micro/Nanochannel}

In case of straight square channel, there were no simulation up to $180 \mathrm{~nm}$ (width and depth) and 398.1 nm length. The same errors "Divergence detected in AMG solver: pressure correction" and "Divergence detected in AMG solver: x-momentum" are occurred. Simulation was done for $180.1 \mathrm{~nm}$ (width and depth) and $398.2 \mathrm{~nm}$ length with the same warning error. Error free simulation takes place for the value of 180 $\mu \mathrm{m}$ (width and depth) and $180 \mu \mathrm{m}$ length. It means that ANSYS has simulation problems in this range with some channels.

\subsection{Simulation of Curvilinear Micro/Nanochannel}

In case of curvilinear channel, no simulation was done up to $50 \mathrm{~nm}$ radius. Same errors "Divergence detected in AMG solver: pressure correction" and "Divergence detected in AMG solver: $x$-momentum" are occurred. Simulation was done for $50.1 \mathrm{~nm}$ radius with the

Mehran University Research Journal of Engineering and Technology, Vol. 39, No. 4, October 2020 [p-ISSN: 0254-7821, e-ISSN: 2413-7219] 
warning error. Simulated length was found for this channel as $829 \mathrm{~nm}$ with the same warning error. In micrometer range error free and trusted simulations were done for $22 \mu \mathrm{m}$ radius and $209.1 \mu \mathrm{m}$ length and beyond.

\subsection{Validation of CFD results}

Dietrich et. al. [30] used straight square millimetric channel for their research and $180 \mu \mathrm{m}$ (width and depth) and $180 \mu \mathrm{m}$ length has been reported in this research. With high aspect ratio, $150 \mu \mathrm{m}$ width and 600 $\mu \mathrm{m}$ length rectangular microchannel was fabricated and tested by Campo and Greiner [31]. This aspect ratio is higher than our simulated aspect ratio. Wewala et. al. [32] used curvilinear microchannel with minimum and maximum inner radius was 100 and $1150 \mu \mathrm{m}$. The authors have reported just $22 \mu \mathrm{m}$ radius and $209.1 \mu \mathrm{m}$ length for better fluid flow.

The literature has no investigations for the least aspect ratio of micro/nano-channels. Researchers have been useing micro/nano-channels with higher aspect ratios. Therefore, validation of CFD results are not possible for all micro/nano-channels. In this study, the authors have simulated micro/nano-channels for better fluid flow.

\subsection{The Issue of Errors}

In these errors, when pressure was changed or corrected then message of x-momentum error was shown on the screen. When momentum was changed or corrected then the message of pressure correction error shown on screen. The other errors were "1 solution is converged", "Warning: convergence tolerance of 1.000000e-6 not reached during Hybrid Initialization" and "GuiOperation Edit". The error message "1 solution is converged" meant no simulation. Fluid could not be entered channel with any desired values of pressure and velocity. The error "Warning: convergence tolerance of 1.000000e-6 not reached during Hybrid Initialization" occurred after the initialization in set up tool of ANSYS. It was investigated that the default number of iterations were not enough and we had to increase the number of iterations and re-initialize the flow. But when number of iterations were corrected then the above said errors occurred at nano meter range. With warning error, the first five iterations are zero in the result. The error when simulation was done without meshing was also very strange. This error occurred for any dimension of nanochannels. However, this type of error could be controlled by using correct mesh. Sometimes this error could not be controlled for a particular geometry and dimension of microchannels. The error of "GuiOperation Edit" occurred when ANSYS software is used again and again for many simulations. In order to get rid of these errors we have to change the geometry or the size of the channel. We cannot simulate our desired micro/nanochannel with desired dimensions in ANSYS.

Moreover, simulation encountered convergence failures when an imbalance geometry was used in ANSYS FLUENT. This failure meant that there was too much imbalance in the system. The calculated reaction forces did not match the applied loads. Although, ANSYS FLUENT tried hard to make the flow and overcome the imbalances, it has not been able to do so and stops. If force residuals under solution information was observed, then it meant that the solver has been unable to get the force convergence residual and imbalance force. Sometimes ANSYS has performed simulation for an imbalance geometry with no mesh or mesh error.

\section{CONCLUSION}

ANSYS FLUENT is used for each and every simulation. Some limitations are found in software simulation of different geometries of micro/nanochannel. Simulation depends upon some suitable aspect ratio of the channels and also on courant number. The simulation with more suitable dimensions of the micro/nano channels does not depend upon courant number. Moreover, simulation cannot be done due to the limitations of the software at nanometer range for different micro/nano-channel. There are total 18 versions of ANSYS but ANSYS Company has not considered this issue. ANSYS Company has not yet enhanced the capacity of simulation with all dimensions in nanometer range. The options of units are also same as eighteen years before. There is no nanometer unit in ANSYS as an option. The channels with all dimensions in nano 
range can be created through design modeler and meshed in mesh analysis. It means the design modeler and mesh analysis tools are very powerful tools. These tools can handle objects of nano size and even below than this. But set up and solution tool of ANSYS is not very powerful. It cannot simulate at this level. ANSYS set up and solution tool should simulate at this level with all dimensions in nano range as its other tools can handle this level. According to the academic aspects, simulation, relating to aspect ratio is the best way to have better fluid flow measurements before the fabrication of all fluidic channels. Additionally, in literature, the aspect ratio of all micro/nano-channels can be found but there is no study for the least aspect ratio for these channels. In this study, the authors have found the least aspect ratio with which simulation can run and after fabrication, the channels will be useful for measuring the flow parameters. Excellent aspect ratio means a better fluid flow through the channel. This research work will be found very useful for those researchers who want to do simulation for their research work.

\section{ACKNOWLEDGEMENT}

This simulated work was performed in parts in The University of Lahore Lab and Government College University, Electronics Lab.

\section{REFERENCES}

[1] Galambos, P.C., Okandan, M., Montague, S., Smith, J.H., Paul, P.H., and Krygowski, T.W.,"Surface Micromachined Microfluidic Devices", Google Patents, January, 2003.

[2] Maenaka, K., "MEMS Inertial Sensors and their Applications", Proceedings of the $5^{\text {th }}$ IEEE International Conference on Networked Sensing Systems, pp. 71-3, 2008.

[3] Calvert, P., "Inkjet Printing for Materials and Devices", Chemistry of Materials, Vol. 13, pp. 3299-305, 2001.

[4] Norton, T., and Sun, D.W., "Computational Fluid Dynamics (CFD) - An Effective and Efficient Design and Analysis Tool for the Food Industry: A Review", Trends in Food Science and Technology, Vol. 17, pp. 600620, 2006.

[5] Van, P.F., Hornbeck, L.J., Meier, R.E., and
Douglass, M.R., "A MEMS-Based Projection Display", Proceedings of the IEEE, Vol. 86, pp. 1687-704, August, 1998.

[6] Sia, S.K., and Kricka, L.J., "Microfluidics and Point-of-Care Testing", Lab on a Chip, Vol. 8, pp. 1982-3, December, 2008.

[7] Marcus, J.S., Anderson, W.F., and Quake, S.R., "Parallel Picoliter RT-PCR Assays Using Microfluidics", Analytical Chemistry, Vol. 78, pp. 956-8, February, 2006.

[8] Sohraby, K., Minoli, D., and Znati, T., "Wireless Sensor Networks: Technology, Protocols, and Applications", John Wiley and Sons, April, 2007.

[9] Baloch, Z.A.K., Qureshi, A.L., Sahito, A.R., and Baloch, A., "Numerical Simulation of Mixing of Effluent through Porous Media: The Effects of Local Inertia on the Flow", Mehran University Research Journal of Engineering and Technology, Vol. 37, pp. 405-16, April, 2018.

[10] Rosaguti, N.R., Fletcher, D.F., and Haynes, B.S., "Low-Reynolds number heat transfer enhancement in sinusoidal channels", Chemical Engineering Science, Vol. 62, pp. 694-702, 2007.

[11] Lee, G.B., Chang, C.C., Huang, S.B., and Yang, R.J., "The hydrodynamic focusing effect inside rectangular microchannels", Journal of Micromechanics and Microengineering, Vol. 16, pp. 1024, 2006.

[12] Ashraf, M. W., Tayyaba, S., Bodhale, D. W., and Afzulpurkar, N., "Design and Simulation of Microchannel for Cell Sorting System," Proceedings of the First International Conference on Manipulation, Manufacturing and Measurement on Nanoscale (3M-Nano), Changchun, China, 29 August-2 September, 2011.

[13] Tayyaba, S., Ashraf, M.W., Khan, M.S., Afzulpurkar, N., and Imran, M., "Sinusoidal Microchannel Simulation for Cell Sorting System", Bahria University Journal of Information and Communication Technology", Vol. 6, pp. 37-42, December, 2013.

[14] Solehati, N., Bae, J., and Sasmito, A.P., "Numerical investigation of mixing performance in microchannel $\mathrm{T}$-junction with wavy structure", Computers and Fluids, Vol. 96, pp. 10-9, June, 2014. 
[15] Özkan, A., and Erdem, E.Y., "Numerical analysis of mixing performance in sinusoidal microchannels based on particle motion in droplets", Microfluidics and Nanofluidics, Vol. 19, pp. 1101-8, August, 2015.

[16] Chiam, Z.L., Lee, P.S., Singh, P.K., and Mou, N., "Investigation of fluid flow and heat transfer in wavy micro-channels with alternating secondary branches", International Journal of Heat and Mass Transfer, Vol. 101, pp. 1316-30, October, 2016.

[17] Suriano, R., Kuznetsov, A., Eaton, S.M., Kiyan, R., Cerullo, G., and Osellame, R., "Femtosecond laser ablation of polymeric substrates for the fabrication of microfluidic channels2, Applied Surface Science, Vol. 257, pp. 6243-50, May, 2011.

[18] Andersson, H., and Vanden, B.A., "Microfluidic devices for cellomics: a review", Sensors and actuators B: Chemical, Vol. 92, pp. 315-25, 2003.

[19] Malic, L., Brassard, D., Veres, T., and Tabrizian, M., "Integration and detection of biochemical assays in digital microfluidic LOC devices", Lab on a Chip, Vol. 10, pp. 418-31, February, 2010.

[20] Nivedita, N., Ligrani, P., and Papautsky, I., "Dean Flow Dynamics in Low-Aspect Ratio Spiral Microchannels", Scientific Reports, Vol. 7, October, 2017.

[21] Damian, I.R., Tanase, N.O., and Balan, C., "Aspect ratio influence on flow focusing in cross microchannels", Proceedings of the 10th International Symposium on Advanced Topics in Electrical Engineering (ATEE): IEEE, pp. 258-61, July, 2017.

[22] Afzal, M.J., Tayyaba, S., Ashraf, M.W., Hossain, M.K., Uddin, M.J., and Afzulpurkar, N., "Simulation, Fabrication and Analysis of Silver Based Ascending Sinusoidal Microchannel (ASMC) for Implant of Varicose Veins", Micromachines, Vol. 8, pp. 278-301, September, 2017.

[23] Kwak, H.J., Kim, J.H., Myung, B.S., Kim, M.H., Kim, D.E., "Behavior of pool boiling heat transfer and critical heat flux on high aspect-ratio microchannels", International Journal of Thermal Sciences, Vol. 125, pp. 111-20, March, 2018.

[24] Afzal, M.J., Tayyaba, S., Ashraf, M.W.,
Hossain, M.K., and Afzulpurkar, N., "Fluidic simulation and analysis of spiral, U-shape and curvilinear nano channels for biomedical application", IEEE International Conference on Manipulation, Manufacturing and Measurement on the Nanoscale (3M-NANO), pp. 190-4, August, 2017.

[25] Afzal, M.J., Ashraf, M.W., Tayyaba, S., Hossain, M.K., and Afzulpurkar, N., "Sinusoidal Microchannel with Descending Curves for Varicose Veins Implantation", Micromachines, Vol. 9, pp. 59-75, January, 2018.

[26] Nadeem, A., Ahsan, K., and Sarim, M., "Illustration, Detection \& Prevention of Sleep Deprivation Anomaly in Mobile Ad Hoc Networks", Mehran University Research Journal of Engineering and Technology, Vol. 36, pp. 233-42, May, 2017.

[27] Memon, F., Memon, A.H., Talpur, S., Memon, F.A., and Memon, R.N., "Design and Co-Simulation of Depth Estimation Using Simulink HDL Coder and Modelsim", Mehran University Research Journal of Engineering and Technology, Vol. 35, pp. 473-82, May, 2016.

[28] Javaid, Q., Arif, M., Talpur, S., Korai, U.A., and Shah, M.A., "An Intelligent ServiceBased Layered Architecture for eLearning and eAssessment", Mehran University Research Journal of Engineering and Technology, Vol. 36, pp. 97-116, May, 2017.

[29] Javaid, Q., Memon, F., Talpur, S., Arif, M., and Awan, M.D., "Mining Frequent Item Sets in Asynchronous Transactional Data Streams over Time Sensitive Sliding Windows Model", Mehran University Research Journal of Engineering and Technology, Vol. 35, pp. 625-44, May, 2016.

[30] Dietrich, N., Loubiere, K., Jimenez, M., Hebrard, G., and Gourdon, C., "A new direct technique for visualizing and measuring gasliquid mass transfer around bubbles moving in a straight millimetric square channel", Chemical Engineering Science, Vol. 100, pp. 172-82, April, 2013.

[31] del Campo, A., and Greiner, C., "SU-8: a photoresist for high-aspect-ratio and 3D submicron lithography", Journal of Micromechanics and Microengineering, Vol. 17, pp. 81, June, 2007. 
[32] Wewala, W., Kasi, J.K., Kasi, A.K., and Afzulpurkar, N., "Design, Simulation and Comparison of Ascending and Descending Curvilinear Microchannels for Cancer Cell Separation from Blood", Biomedical Engineering: Applications, Basis and Communications, Vol. 25, pp. 1350037, April, 2013. 